\title{
Causes and consequences of a lack of coevolution in Müllerian mimicry
}

\author{
JAMES MALLET \\ Galton Laboratory, Department of Biology, University College London, 4 Stephenson Way, \\ London NW1 2HE, England \\ (http://abacus.gene.uel.ac.uk/jim/)
}

Received 9 June 2000; accepted 27 November 2000

Co-ordinating editor: C. Rowe

We are rarely able in such investigations to arrive at entirely satisfactory conclusions owing to lack of adequate material and data, and I fear the present effort is no exception. The results may, however, serve to indicate the directions in which future workers ... may hope to obtain more definite results (Eltringham, 1916).

\begin{abstract}
Müllerian mimicry, in which both partners are unpalatable to predators, is often used as an example of a coevolved mutualism. However, it is theoretically possible that some Müllerian mimics are parasitic if a weakly defended mimic benefits at the expense of a more highly defended model, a phenomenon known as 'quasi-Batesian mimicry'. The theory expounded by Müller and extended here for unequal unpalatability, on the other hand, suggests that quasi-Batesian mimicry should be rare in comparison with classical, or mutualistic Müllerian mimicry. Evolutionarily, quasi-Batesian mimicry has consequences similar to classical Batesian mimicry, including unilateral 'advergence' of the mimic to the model, and diversifying frequency-dependent selection on the mimic which may lead to mimetic polymorphism. In this paper, theory and empirical evidence for mutual benefit and coevolution in Müllerian mimicry are reviewed. I use examples from wellknown insect Müllerian mimicry complexes: the Limenitis-Danaus (Nymphalidae) system in North America, the Bombus-Psithyrus (Apidae) system in the north temperate zone, and the HeliconiusLaparus (Nymphalidae) system in tropical America. These give abundant evidence for unilateral advergence, and no convincing evidence, to my knowledge, for coevolved mutual convergence. Furthermore, mimetic polymorphisms are not uncommon. Yet classical mutualistic Müllerian mimicry, coupled with spatial (and possibly temporal) variation in model abundances convincingly explain these apparent anomalies without recourse to a quasi-Batesian explanation. Nevertheless, the case against classical Müllerian mimicry is not totally disproved, and should be investigated further. I hope that this tentative analysis of actual mimicry rings may encourage others to look for evidence of coevolution and quasi-Batesian effects in a variety of other Müllerian mimicry systems.
\end{abstract}

Key words: advertising coloration, aposematism, Batesian mimicry, mimicry, mutualism, signalling, warning colour 


\section{Introduction}

Traditional Müllerian mimicry, in which unpalatable species copy one another for mutual benefit, is one of the longest-recognized and best studied mutualisms. Many butterflies, Hymenoptera, other insects, or even vertebrates such as fish or coral snakes (Wickler, 1968; Edmunds, 1974; Turner, 1977; Joron and Mallet, 1998), belong to Müllerian mimicry 'rings' within a local area. The advantages of Batesian mimicry, in which a palatable mimic parasitizes an unpalatable model by copying its warning colour pattern, are fairly obvious. In contrast, Müllerian mimicry, in which both partners are unpalatable, has been less easy to accept. Even the inventor of mimicry, Bates (1879) had little good to say about Müller's (1879) paper when first read at the Entomological Society of London. Today, Müller's insight retains a strong following (e.g. Wickler, 1968; Edmunds, 1974; Turner, 1977, 1984; Joron and Mallet, 1998; Mallet and Joron, 1999), but is under renewed attack from a variety of quarters (Owen and Owen, 1984; Huheey, 1988; Speed, 1993; Speed and Turner, 1999).

In what follows, I discuss theoretical causes and evolutionary consequences of traditional, mutualistic Müllerian mimicry, and I attempt to glean evidence for or against coevolution, in the strict sense of mutual evolutionary convergence, from empirical and comparative data. It has recently been suggested that 'the end of traditional Müllerian mimicry' may be at hand (Speed, 1993), and that an intermediate parasitic form of Müllerian mimicry known as 'quasiBatesian mimicry', may be abundant (Speed and Turner, 1999); these ideas are discussed below in the section 'Mutual Benefit'. Even without quasi-Batesian complications, Müllerian mimicry remains both frustrating for those who would like a simple story, and a delight to the specialist. The more one investigates theory and examples of mutualistic mimicry, the more complex and less clear this deceptively simple adaptation becomes.

\section{The nature of Müllerian mimicry and its consequences}

Associated with Müllerian mimicry are a number of theoretical distinctions which may or may not apply in every case. These are (A) unprofitability of both model and mimic, (B) purifying ('positive') number- and frequency-dependent selection, (C) mutual benefit, (D) mutual evolutionary convergence, and (E) phylogenetic co-divergence. I discuss each in turn, and comment on their role in the concept originally outlined by Müller (1879).

(A) Unprofitability of both model and mimic. In Müllerian mimicry, both partners are unprofitable. Unprofitability means that predators can learn to avoid the species in the absence of any mimics. Unprofitability includes 
unpalatability, as well as other punishment, such as stings, disgusting smells, nasty bites, or even rapid escaping ability, so that the costs of pursuit and handling outweigh any benefits of prey capture. Hereafter, the more traditional term 'unpalatability' should be taken to mean any kind of unprofitability. Unprofitability may of course depend on the condition of the prey, the species of predators and their hunger levels, but mimicry theory generally assumes that 'unpalatable' prey are far from the profitable/unprofitable threshold, so that their unprofitability holds in most circumstances. After experience with some members of an unprofitable species, the predator must be able to recognize other members of that same species and any mimics, resulting subsequently in a lower attack probability. Only if both partners are unprofitable in this sense can mimicry be said to be Müllerian (see also Speed and Turner, 1999).

(B) Purifying frequency-dependent selection. Unprofitability leads to an interesting form of frequency-dependent selection known as 'number-dependent selection' (Turner, 1978; Mallet and Joron, 1999). As the predator learns to avoid the prey, the number attacked quickly plateaus when prey encounters rise above some threshold (Fig. 1A), so that the fraction of prey eaten during predator learning declines hyperbolically with density (Fig. 1B). This response to prey density is known in ecology as a 'Type II' functional response, and occurs in many simple predator-prey systems where predators become satiated (e.g. see Ricklefs and Miller, 2000). A major difference between unprofitable and profitable prey is that predator satiation is due to active avoidance, and occurs at a very low population density - by definition, it is very unlikely that unprofitable prey can ever form a major part of the diet. Another difference is that intelligent predators will switch to profitable prey as they become more abundant, resulting in a rising fraction of prey attacked, at least initially (a 'Type III' functional response, Fig. 1). Thus, predator functional responses to unprofitable prey differ both quantitatively, in having a much lower plateau, and qualitatively, in lacking an inflection point, from those to profitable prey.

Now suppose two equally unpalatable colour morphs occur within a prey species. Then predation on each morph leads to number-dependent selection: the rarer morph within a species suffers a similar number, and therefore a greater fraction, of attacks during predator learning, and is selected against. (The effect depends on the morphs being separately learned; to the extent that the morphs are confused by predators, selection will be reduced.) This kind of frequency-dependent selection, usually called 'positive' or 'purifying' frequency-dependent selection is strongest against the rarer morph, leading to unstable polymorphisms and eventual monomorphism as an evolutionary result. As a fundamental result of distinction (A), 

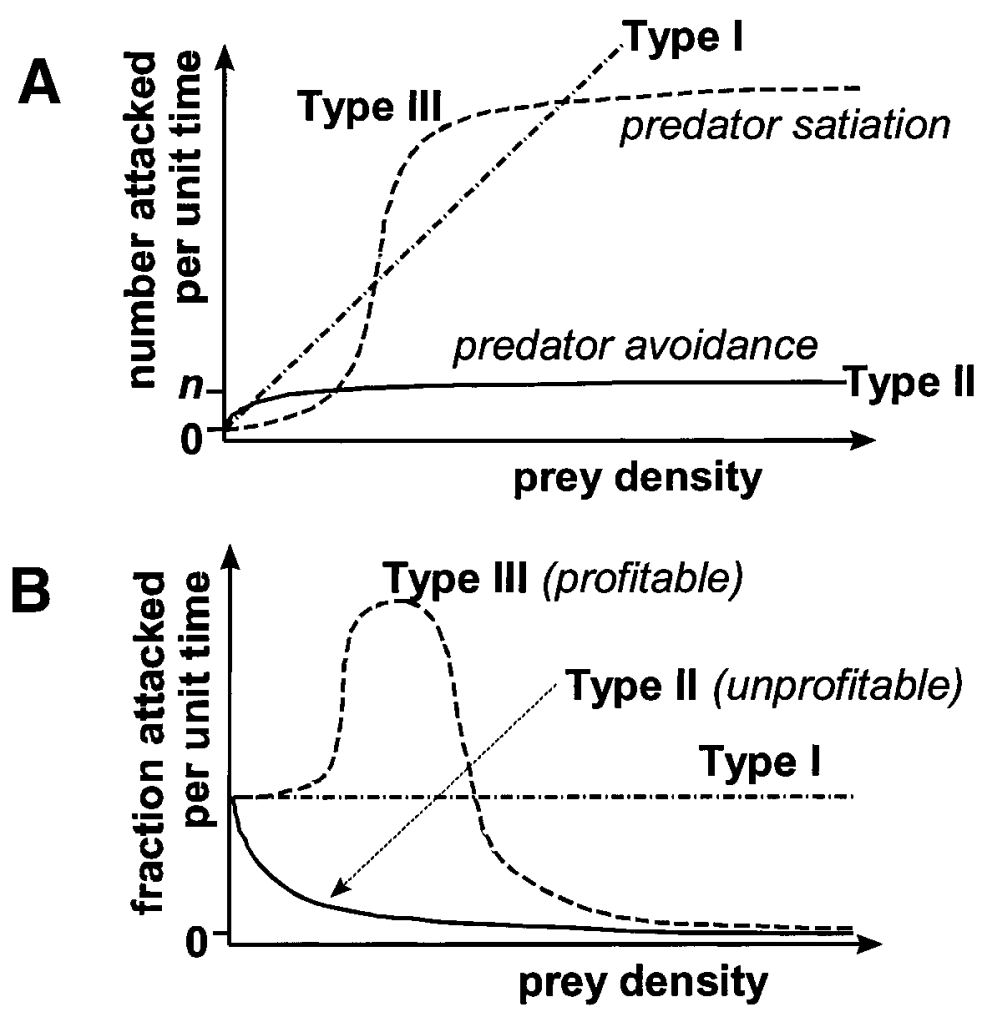

Figure 1. Functional responses of predators to prey of varying profitability. (A) Functional responses of predators in terms of total number attacked per unit time. (B) Functional response in terms of fraction attacked per unit time. Type I responses are a characteristic of models producing quasi-Batesian mimicry. Classical Type II responses with a very low number attacked are characteristic of avoidance learning under traditional Müllerian assumptions. The asymptote $n$ in $\mathbf{A}$ represents the Müllerian palatability parameter (Müller, 1879), equivalent to $n_{k}$ in Mallet and Joron (1999). Finally, Type III responses are expected when predators initially learn to attack palatable prey, but then become satiated at high prey densities. Is important to realize that 'fraction attacked per unit time' assumes that learning and forgetting by predators has equilibrated in a given time period; it measures the total cost of predator learning on the prey population; it is the same as the 'asymptotic attack fraction' in Mallet and Joron (1999). Confusingly, the learning saturation asymptote at any density assumed in the term 'asymptotic attack fraction' is not the same the asymptotic attack fraction of zero reached with high prey density, as shown in the graphs.

intraspecific purifying frequency-dependent selection should occur within all unpalatable species. If a predator confuses two unpalatable species, this purifying selection also acts across the species boundary, as in Müllerian mimicry.

(C) Mutual benefit. If both species are unpalatable and their colour patterns are confused by predators, we may say, following (A) that they are effectively 
Müllerian mimics (effectively, because similarity of pattern may not be adaptive, though it almost always will be in nature). A combination of two such indistinguishable species, each under purifying number-dependent selection, might be expected to exhibit the feature that, as we add individuals of either species to the local population, the total attack rate on each will be reduced. In Müller's (1879) theory (reprinted in Joron and Mallet, 1998), this is so, because predators learn by taking a certain number of individuals of either species within a season, and thereafter avoid prey with that colour pattern. Any further additions to the population will reduce the per capita attack rate. Müller's own theory treated only the case of equal unpalatability, but can be extended for variable levels of defence. Provided both species are unprofitable and avoided, both species benefit to some extent, whatever their relative numbers or palatabilities (see Appendix).

This Müllerian theory results in an approach to an asymptotic fraction attacked of zero (Fig. 1B), because as prey density is increased the fixed numbers attacked per unit time become progressively diluted. However, after considering a variety of theories of predator 'memory dynamics', Speed (1993) and Speed and Turner (1999) criticized the Müllerian assumption, suggesting instead that the fraction attacked may reach a non-zero asymptote with prey density (a 'Type I' functional response lacking in predator satiation, Fig. 1). Then the asymptotic fraction attacked of a combination of similar mimics should be some average of asymptotic fractions due to each species alone, and a less unpalatable species would have a deleterious effect on the asymptotic attack rate of the more unpalatable species. Although the mimicry would be Müllerian in the sense that both species can reinforce a lower-than-naive predation fraction on their own, the more palatable species will gain and the less palatable will lose from this type of Müllerian mimicry, once learning saturation is reached. This potential for a parasitic form of Müllerian mimicry has been termed 'quasi-Batesian mimicry' (Speed, 1993; Speed and Turner, 1999).

Quasi-Batesian mimicry seems particularly attractive because it should cause diversifying frequency-dependent selection, and could lead to a mildly unpalatable species becoming a stable polymorphic mimic of multiple unpalatable models. Mimetic polymorphisms do indeed exist in unpalatable species such as bumblebees and Heliconius (see below). Traditional Müllerian theory predicts only mutual benefits and purifying selection, so that polymorphisms would be unstable, and it has generally been assumed that classical Müllerian mimicry cannot explain such polymorphisms. However, it is one of the purposes of this paper to demonstrate how readily spatial and temporal heterogeneity can explain mimetic polymorphisms in unpalatable species under mutualistic Müllerian mimicry (see also Brown and Benson, 1974; Plowright and Owen, 1980; Mallet and Joron, 1999; Kapan, 2001; Joron et al., this issue, and see 
below under Heliconius). Thus, while quasi-Batesian mimicry may lead to polymorphisms, the logic is not reversible: the existence of polymorphic Müllerian mimics does not require quasi-Batesian mimicry as an explanation.

I am somewhat skeptical of quasi-Batesian mimicry theory (Joron and Mallet, 1998; Mallet and Joron, 1999) because of potential flaws in the theories of memory dynamics used by Speed; indeed Speed now agrees with some but not all of our critique (Speed, this issue). In Müllerian theory, the asymptotic attack rate on each species alone is zero, and indeed it doesn't seem very likely that under any reasonable theory of learning that the functional response to unpalatable prey will be other than Type II (Fig. 1). A Type II response with a zero attack fraction asymptote is expected for predator satiation, even without any avoidance learning at all, and is arguably the most general type of predator functional response; avoidance of unpalatable prey differs mainly in that the attack rates will rarely be as high as for palatable prey. In contrast, recent experiments with birds using quinine/pastry baits in the field (Speed et al., 2000) and laboratory (S. Hannah et al., in preparation) suggest that the empirical basis of quasi-Batesian mimicry may be on firmer ground than the theory hitherto used to explain these results. In these recent experiments, predator experience with less unpalatable baits can result in increasing attacks on more unpalatable baits. Weakly unpalatable baits are therefore parasitic mimics of strongly unpalatable ones, even though both are increasingly avoided when presented on their own. The authors argue that these results disprove the idea that predator learning will always result in a number- (or dose-) dependent, Type II functional response. However, it is also possible that the taste disincentive of the most distasteful pastry is so weak that birds risk little by pecking them on the off chance that they may be palatable. The problem with Müllerian theory may have little to do with memory dynamics, but with an innate tendency to experiment where the costs of ignoring memory are low. This controversy over quasi-Batesian mimicry is debated more fully elsewhere (Mallet and Joron, 1999), and will not be discussed further here. However, I do agree that the newer empirical results are intriguing and require further attention. In any case, although I believe quasi-Batesian mimicry theory to be an artefact of an unrealistic memory algorithm, I agree with Speed and Turner (1999) that its theoretical and practical possibility means that Müllerian mimicry, as defined in (A) and (B) does not necessarily imply mutual benefit.

(D) Coevolution: mutual evolutionary convergence. It is perhaps natural to assume that strict coevolution, in this case mutual convergence, is a characteristic of Müllerian mimicry (e.g. Dixey, 1907, 1909). Under Müller's (1879) theory, although both species always benefit to some extent, the ratio of mimetic benefits is the square of the relative abundances for a pair of species with 
equal unpalatability. This is also true for differences in unpalatablity: 'protection' is a product of unpalatability and abundance (see Appendix). Thus, if species $\alpha$ is three times as common or three times as chemically protected as its Müllerian mimic $\beta$, the latter gains nine times as much benefit from mimicry as $\alpha$.

Even if the benefits are ultimately mutual, it is probable that Müllerian mimicry can evolve initially in only one of the partners. Lop-sided benefits will generate a unilateral form of evolution, called 'advergence' (Turner, 1995) to distinguish it from strictly bidirectional mutual convergence. Suppose, as in Müller's assumption, both species are equally unpalatable and initially look different. A perfectly mimetic mutant in the rarer species is always favoured, while a similar mutant in the commoner species loses the protection of its own species, and gains only the weaker protection of the rarer species (Marshall, 1908; Turner, 1977; Sheppard et al., 1985). The rarer species is the 'mimic' and the commoner is the 'model' in terms of evolutionary trajectory, even though there will be mutual benefit between 'co-mimics' once mimicry has evolved. Once again, a similar argument applies to pairs of species which are unequally protected chemically, as well as numerically, so that it is not always the most common species that is the model. Because dosage underlies the predator response to unpalatability as well as to density, the model will be the species with the maximal product of abundance and unpalatability (see Appendix). Thus, there are two reasons why mimicry will evolve unidirectionally: firstly, because the ultimate benefit is much greater to the less protected species; secondly, because of initial frequency-dependent selection against mimicry in the more protected species.

Müllerian mimicry therefore has a kind of non-Euclidean mutual adaptive surface with respect to the numbers of different patterns in each pair of comimics. For the more protected species, the adaptive surface consists of a pair of adaptive peaks: a lower adaptive peak corresponding to fixed non-mimicry, and a somewhat higher fitness peak corresponding to the mutual benefits of mimicry, separated by a trough of low fitness with intermediate frequencies of mimetic and non-mimetic morphs. For the less protected species, in contrast, the adaptive surface slopes smoothly upward towards a single fitness peak of 100 percent mimicry, and non-mimicry is unstable to mimetic mutations. The distinction between ultimate mutualism and bidirectional coevolution of Müllerian mimics was first clearly enunciated by Marshall (1908); more recently, the theory has been admirably clarified and updated by Turner (1977, 1984) and Sheppard et al. (1985).

So far, I have treated mimicry as though it arises as a single, perfectly mimetic mutation. This is usually very far from the truth. Quantitative genetic theories of Müllerian convergence lead to still more interesting evolutionary curiosities of mimicry evolution. If two unpalatable species look very different, 
intermediate steps towards mimicry may be disfavoured because they fall between two stools: imitation of the model is poor, and purifying selection acts against deviants from the ancestral pattern. Mimicry is likely to be favoured only if mutation provides a major leap towards the model, so that the mimetic benefit outweighs the loss of protection of the species' own colour pattern protection. This argument against gradual evolution of mimicry was briefly touched upon by Marshall (1908), but subsequently improved by others. In the words of John R.G. Turner, there is an 'evolutionary sieve' which prevents gradual evolution of mimicry, and selects for more radical mutations (Nicholson, 1927; Turner, 1976, 1984). Of course, perfect mimicry is unlikely to arise via a single mutation: once reasonable mimicry is achieved, mutual convergence can take place. Turner (1984) calls this a 'two-step' theory of Müllerian mimicry. In step 1, Müllerian advergence takes place via a few major allelic steps, and gradual mutual convergence is impossible initially; in step 2, once the two forms are approximate mimics, an intermediate pattern can be more favourable than either extreme, and gradual, mutual, and polygenic convergence can take place (Turner, 1984; Sheppard et al., 1985). Exactly how two-step evolution meshes with actual predator perception of colour patterns is hard to say. Two patterns appearing radically different to us may even have hidden dimensions along which gradual mutual convergence can 'tunnel', leading to a collapse of the need for step 1, although this seems unlikely.

Müllerian mimicry between Heliconius erato and $H$. melpomene has sometimes been used to supply examples of mutualistic coevolution (Gilbert, 1983; Thompson, 1994). If the Müllerian theory hitherto outlined is correct, coevolution can only take the limited form of 'alternating resemblance' (Marshall, 1908), where the first species may adverge to the second in regions where the second species is more protected, while the second species may adverge to the first in other regions where the first is more protected (see also Dixey, 1909; Gilbert, 1983). I discuss these and other examples below and argue that noncoevolutionary explanations are more likely, although it is possible that some alternating coevolution occurs.

(E) Coevolution: phylogenetic co-divergence. The word 'coevolution' might be used in a variety of senses. One definition is 'reciprocal genetic change in interacting species due to natural selection imposed by each on the other' (Futuyma, 1998). Mutual convergence $(D)$ is an example of coevolution in this strict sense. On the other hand, partners may also coevolve in a sense if both respond to the same evolutionary pressures at a similar time, resulting in identical phylogenetic patterns of divergence. This will be especially true if both are also Müllerian co-mimics; the two partners may not be the catalysts of each others' evolutionary direction, but they still affect each others' fitness and so contribute to the overall evolutionary interaction. Thus, it has been suggested 
(Turner, 1984) that the Müllerian mimics Heliconius erato and H. melpomene were isolated in remnant patches of forest during the last ice age, and that this led to 'co-raciation' or similar patterns of geographic colour pattern divergence within each species, both of which were adapting to external pressures, i.e. other species within the same local mimicry rings (e.g. Brown et al., 1974; Turner, 1984). However, this 'Pleistocene Refuge Theory' is no longer universally supported (Mallet, 1993; Turner and Mallet, 1996; Joron and Mallet, 1998), and recent studies of mtDNA phylogeny in both species (Brower, 1996) show that racial genealogies within the two species differ topologically. One of the species, probably $H$. melpomene, has apparently radiated onto pre-existing colour pattern races of the other (Brower, 1996; Mallet et al., 1996; Joron and Mallet, 1998). Müllerian mimicry may yet be a good 'model for coevolution' (Thompson, 1994; Turner, 1995), but there is convincing evidence neither for mutual convergence, nor for coordinated, contemporaneous divergence among co-mimics.

In conclusion, the basis of Müllerian mimicry is that both partners are unpalatable or otherwise unprofitable (A), and that this should lead to purifying number-dependent selection within each species (B). However, these stipulations do not necessarily ensure that the two species are mutualists $(\mathrm{C})$, although in my view Müller's mutualistic outcome is most likely. Coevolution seems likely in most close interspecific associations, but this may not be true for Müllerian mimicry. This form of mimicry may lead neither to mutual convergence (D) nor to contemporaneous divergence due to similar causes in both co-mimics (E). Existing theory suggests that most Müllerian mimicry will mainly be achieved by one-sided advergence by a mimic onto a model's colour pattern, as in Batesian mimicry.

\section{Empirical evidence for directionality of mimicry evolution}

'Which is the model and which is the mimic?' This is probably the most frequently asked question after lectures on Müllerian mimicry. The classic answer is that Müllerian co-mimics play both roles, that they benefit one another (provided mimicry is not quasi-Batesian). However, if Müllerian mimicry normally evolves via advergence, rather than coevolution, we might expect to find evidence that some members of mimicry rings are models, while others are mimics which adverge to them, exactly as in Batesian mimicry. The remainder of this article tentatively explores experimental and comparative evidence for and against coevolution, and, if advergence is found likely, for evidence of which partners are models and which are mimics. I believe that this is the first attempt at a synthesis of this nature for Müllerian mimicry, but I do not claim originality because most or all of the ideas herein surface as implicit or explicit 
assumptions in the literature on particular mimicry systems (e.g. Bates, 1862; Marshall, 1908; Eltringham, 1916; Plowright and Owen, 1980; Turner, 1995). I give a few examples from insect mimicry rings, although I recommend a general search for other, maybe better examples from a variety of terrestrial and marine mimicry systems.

What kinds of evidence from Müllerian mimicry rings might then suggest which species are the models? Below I outline a number of ideas, many taken from the literature on Batesian mimicry (e.g. Edmunds, 1974; Turner, 1995). Species which are models in Müllerian mimicry rings might be expected to be:

(1) More unpalatable,

(2) Commoner,

(3) Earlier (in seasonal species),

(4) Larger,

(5) More conspicuous,

(6) More gregarious,

... and should have:

(7) A wider geographic distribution,

(8) Clearer and less fuzzy colour patterns (mimics should be 'impressionistic' copies),

(9) More ancient colour patterns,

(10) Less polymorphism, and

(11) Less overall divergence from an ancestral colour pattern ... than their mimics.

In case I have left something out, these ideas categorize into the causes of one-sided mimicry, that is the greater apparency and unpalatability of the model (1-7), and the phylogenetic consequences of more recent radiation by mimics (8-11). These are not intended to be hard and fast rules; instead they are generalized tendencies. For example, Laparus doris is almost certainly a mimic, but it is an exception to rule (6) in that its larvae are the most gregarious of all heliconiines, and quite possibly the most gregarious known in any butterfly (see below). The causes of one-sided advergence should by now be fairly obvious, but the phylogenetic consequences require some discussion. That mimicry is an 'impressionistic' copy of an original model pattern (8) may often be very difficult to pin down; nevertheless, there are some very good examples in presumed Batesian mimics, and we can expect similar examples to turn up in Müllerian systems. For example, the pigmented costal margin of the wings of many dipteran Batesian mimics is clearly an impressionistic imitation of a structurally anomalous feature of wasp wings: many social wasps simply happen to fold the anterior portion of their wings when not flying, creating a more opaque and therefore visually browner costal margin which mimics copy by using pigment (Waldbauer, 1988). The fact that mimic colour patterns must by definition evolve after the model pattern has diverged (9) means that groups 
of organisms that are predominantly mimics will tend to be differentiated in pattern at a lower taxonomic rank than their models, as species compared with genera of their models, say (Marshall, 1908; Eltringham, 1916), or as polymorphisms or geographic races within species (10), compared with good species of models. There will therefore be a tendency for phylogenetic groups of mimics of to adopt a greater diversity of colour patterns than phylogenetic groups of equivalent rank which predominantly acting as models (11, see also Marshall, 1908; Eltringham, 1916).

I conclude this discussion with three examples of mimicry systems that display various combinations of these traits.

The monarch-queen-viceroy story

Data on unpalatability of mimics in nature is perhaps the hardest information to obtain about mimicry. For a long while, it was supposed that the monarch Danaus plexippus, the queen D. gilippus and the rarer D. eresimus butterflies were models for Batesian mimicry by the North American viceroy Limenitis archippus. Recently it has been shown, using hand-reared red-winged blackbirds Agelaius phoeniceus as predators, that the viceroy is about as unpalatable as the monarch, and more unpalatable than the queen, its supposed model in Florida (Ritland and Brower, 1991; Ritland, 1991; but see Brower, 1958, for earlier evidence for unpalatability of Limenitis archippus to a jay). It therefore appeared that D. gilippus, at least in the southern USA, might be either a Batesian mimic or a weakly unpalatable Müllerian mimic of L. archippus and D. plexippus (Ritland, 1991).

However, given the many years of studies showing unpalatablity of Danaidae (summarized by Brower, 1984), it is almost inconceivable that these species are not all Müllerian mimics (Ritland and Brower, 1991; Ritland, 1991). While the palatability data proves that L. archippus is a Müllerian rather than a Batesian mimic, its greater unpalatability than the queen does not necessarily make it a model for the group. Comparative phylogenetic arguments can be brought to bear. Monarchs and queens belong to an ancient genus with colour patterns similar to those occurring worldwide in other Danaus spp. (Ackery and Vane-Wright, 1984). In contrast, a close relative of the viceroy is the redspotted purple (L. arthemis astyanax) a completely different-looking form which joins another North American mimicry ring centred around the iridescent blue-black swallowtail Battus philenor. The two Limenitis are extremely closely related: they hybridize occasionally where they co-occur in the wild (Platt, 1983; Ritland, 1990), and the inheritance of mimicry between the two species can be studied by backcrossing male hybrids (Platt, 1983). Other members of the genus Limenitis are known as white admirals, and are black with white medial bands. These other species are also sufficiently closely related 
to hybridize, including with the mimetic species. Indeed, the northern subspecies L. arthemis arthemis of the mimetic astyanax has this ancestral, nonmimetic pattern (Platt, 1983).

In the northern part of its range, away from the influence of the queen, the viceroy takes on the paler orange colour of the ubiquitous monarch, while in Florida the viceroy is a dark brick red, like D. gilippus and D. eresimus. Its greater geographic divergence (10) and greater difference from the ancestral colour pattern (11) thus strongly suggests that the viceroy mimics Danaus spp. rather than the other way round. Phylogenetic evidence therefore suggests that mimetic patterns in Limenitis have recently diverged from ancestral colours, while those in Danaus are more stable and ancestral (9). The monarch occurs south at least to the Amazon, and, via a sister species D. erippus, the same pattern occurs south to Argentina. The queen is also distributed widely in tropical and subtropical America, while the viceroy barely enters Mexico. Danaus spp. have a wider distribution, again as expected if they were the models (7). Danaus are larger (4), and in my experience, they are also much more common and widespread locally (2). Whatever its palatability, Limenitis is thus almost certainly the advergent mimic. The early and erroneous assumption of Batesian mimicry is probably based on an unconscious appreciation of the comparative and phylogenetic evidence for advergence I have just presented - in its evolutionary trajectory, the viceroy has in fact behaved very like a Batesian mimic.

\section{Bumblebees}

Bumblebees (Bombus spp.) and their relatives are among the most obvious warningly coloured, putatively mimetic organisms in north temperate latitudes. Plowright and Owen (1980) and Williams (1991) convincingly demonstrate colour-pattern convergence among groups of species from the West and East coasts of North America, Western Europe, and Kashmir. In each area, there are at least 1-4 major colour pattern groups or 'mimicry rings'. The resemblances between species give good evidence for convergent (or 'advergent') mimicry rings, since similarities of pattern are stronger between sympatric, unrelated species than between allopatric, related forms. Workers and queens of all species possess a painful sting, while the drones are stingless, and may be Batesian 'automimics' of the females of their own species.

In North America, the species Bombus rufocinctus is polymorphic. A red and yellow morph of this species mimics Bombus ternarius; a black and yellow morph mimics $B$. vagans. The polymorphic mimic emerges later from hibernation than its putative models, and Plowright and Owen (1980) suggest that by the time $B$. rufocinctus emerges, predators will have learnt completely to avoid both colour patterns, making polymorphism nearly neutral where both 
models occur. Spatial and possibly temporal variation in model abundance will then allow maintenance of the polymorphism. Mimicry where the mimic emerges later in season than its model has been termed 'serial mimicry' (Plowright and Owen, 1980). Thus, the later emergence of B. rufocinctus (3) coupled with its mimetic polymorphism (10) argues that this species has converged on the other two, that is, that B. rufocinctus is the mimic (Plowright and Owen, 1980).

The parasitic cuckoo bees of the genus Psithyrus [sometimes sunk within the genus Bombus - see Williams $(1985,1994)$ - but here maintained separate for convenience] attack and kill bumblebee queens and take over their nests, forcing bumblebee workers to rear Psithyrus broods. Psithyrus also join bumblebee mimicry rings. Of highly host-specific species in Europe, Psithyrus rupestris is closely similar to its host $B$. lapidarius, while $P$. barbutellus, $P$. vestalis and $P$. bohemicus are also somewhat mimetic of their hosts, $B$. hortorum, B. terrestris, and B. lucorum, respectively. It used to be thought that each parasite evolved via social degeneration in populations of the host, but it is now known that colour pattern similarity is not due to common ancestry, in part because colour variation of B. lapidarius is followed geographically by variation of its parasite $P$. rupestris across Europe and Asia. Although closely enough related to their hosts to be considered a part of the same genus, genetic and morphological phylogenies show that Psithyrus species form a monophyletic group within Bombus (Plowright and Owen, 1980; Williams, 1985, 1994).

Psithyrus queens sting as effectively as Bombus, and there is no reason to doubt that they are as unpalatable to predators. However, they emerge from hibernation much later (3), usually after the bumblebees have reared a generation of workers. Psithyrus do not produce workers and so must of necessity be less gregarious (6), less common overall (2) and less geographically widespread (7) than their obligate hosts. These Psithyrus almost certainly mimic their hosts rather than vice-versa.

The spring emergence of dipteran Batesian mimics, which occurs 1-3 months before the peak abundance of their hymenopteran models (including Bombus) seems to compromise this 'serial mimicry' hypothesis. The peak fledging period of naive birds also occurs at around the time of the summer peak of hymenopteran density, long after the dipteran mimics have peaked. Waldbauer (1988) argues from these observations that predators learn the models' colour patterns during fledging, the year before they encounter the rarer syrphid or other mimics in the following spring, and cites abundant evidence that predators do indeed remember prey colour patterns over such long periods. I am not expert enough to know whether Waldbauer's serial mimicry argument is an improvement on Plowright and Owen's, but Waldbauer's ideas do seem to cast doubt on the naive idea that models should 
always appear before mimics within a year. The predator should still, of course, encounter the model earlier than the mimic, but this might be at any time in the previous year rather than earlier in the same year. Nonetheless, in Bombus and Psithyrus, the other inferences from relative abundance, distribution, and polymorphism remain convincing. In particular, the cuckoo bees Psithyrus remain excellent candidates for advergent Müllerian mimics of their more abundant and widespread hosts, rather than vice-versa.

\section{Heliconius butterflies}

(a) History The heliconiine butterflies (which Bates called 'acraeoid Heliconidae'), along with the ithomiines and their dismorphiine mimics, are the organisms in which the original discovery of evolutionary mimicry was made (Bates, 1862). Bates is usually credited only with the discovery of Batesian mimicry, but he also noticed mimicry between rare unpalatable heliconiines (such as Heliconius numata - see Joron et al., this issue) and common unpalatable ithomiines. He explained this as mimicry in which the greater rarity of heliconiines led to mimetic selection, in spite of the unpalatability of both. Under definition A, above, we must admit that Bates discovered Müllerian as well as Batesian mimicry (Mallet et al., 1998a) 17 years before Müller published his theory (1879). Bates recognized that the heliconiines were unpalatable because patterns existed in this group that were not mimetic of ithomiines, but which were themselves 'the objects of mimicry' (i.e. models) for other species. Bates was, however, puzzled by similarity of colour patterns between pairs of relatively common unpalatable species, and attributed much of the Müllerian mimicry he saw to co-divergence (see E above) to a common abiotic environment.

In the early 20th century, detailed work on structural morphology led to a reassessment of the heliconiines, culminating in Eltringham's (1916) major systematic analysis of the evolution of mimicry within the genera Heliconius and Eueides. On the basis of male genitalic and androconial (sex scale) morphology, he arranged the genus Heliconius into two major groups: group I, consisting of what I will here refer to as the 'melpomene group'; and group II, consisting of the 'erato group' together with a somewhat more heterogeneous 'basal group'. Eltringham argued that the melpomene group mimicked the erato group, rather than vice-versa, on the basis of a variety of comparative evidence. Firstly, the melpomene group had more intergeneric mimicry than the erato group, while the erato group mainly exhibited intrageneric mimicry (11). For instance, many of the 'silvaniform' subgroup of the melpomene group mimic ithomiines of the genera Melinaea, Mechanitis, Tithorea and Elzunia; even though other members of the group have purely Heliconius-mimicking patterns. Secondly, Eltringham found that single species from the melpomene 
group sometimes contained multiple patterns mimicking a number of species of the other group $(9,10)$. A major example was that $H$. melpomene (within which he included $H$. cydno) mimicked multiple members of the erato group, for example H. erato, $H$. sapho, and $H$. hewitsoni. Thirdly, mimics from outside Heliconius tended to mimic the colour patterns of the erato group more closely than the patterns of their co-mimics in the melpomene group. Eueides tales, pierine butterflies and pericopine moths, for example, mimic the simple rayed pattern of the erato and basal groups, rather than the 'nail-head' rayed pattern (Fig. 2) of the melpomene group, as found in H. melpomene, H. elevatus and $H$. timareta. This provides evidence that the erato group species are the models for intra- and inter-generic mimicry, while the nail-head rays of the melpomene

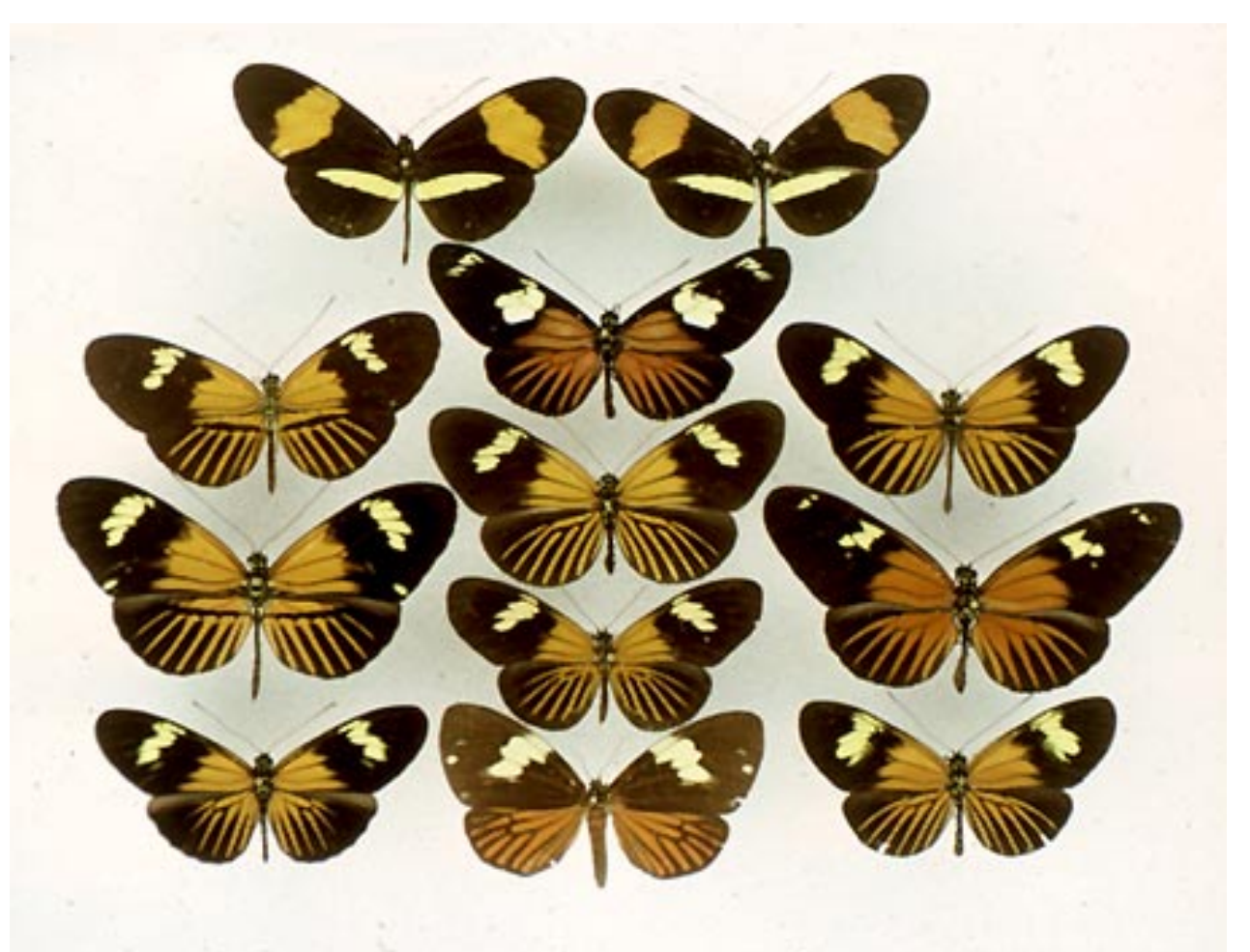

Figure 2. Mimicry rings in Tarapoto, NE Peru. The photo shows Müllerian mimicry (mimetic resemblance in which all the species are unpalatable) between Heliconius erato (left) and $\mathrm{H}$. melpomene (right), and co-mimics in San Martín, Eastern Peru. On the top line are H. erato and $H$. melpomene from the Ríos Mayo and upper Huallaga. Their pattern switches to join a 'rayed' mimicry ring in the lower Huallaga; left - H. melpomene, H. elevatus, H. demeter; centre - Laparus doris (red morph), Neruda aoede, Eueides tales and a pericopine moth; right - H. erato, H. burneyi, and $H$. xanthocles. The two species at top left of the rayed block, $H$. melpomene and its close relative $H$. elevatus have the 'impressionistic' nail-head rays mentioned in the text. Similarly, Laparus doris (top centre of rayed block) also has impressionistic mimicry, in that it has a pair of transverse yellow forewing bands; most potential models have only one (apart from H. burneyi, which is rare, and therefore probably not a model). Photo (c) James Mallet. 
group species are impressionistic copies (8). Finally, the melpomene group species were usually (though not always) rarer (2) and less widespread (7) than the species of equivalent pattern in the melpomene group.

(b) Modern work Eltringham's (1916) work, published during the First World War, was based entirely on museum specimens. Recent systematic work coupled with extensive field and laboratory studies has revolutionized the systematics of Heliconius (Emsley, 1964, 1965; Brown, 1979). With the exception of some troublesome allopatric forms like $H$. pachinus, $H$. timareta, and $H$. heurippa (these are probably no more than strongly divergent races of H. cydno), the species-level taxonomy of the group now appears relatively stable. Some of the taxa recognized by Eltringham as forms of a species are now separated: $H$. cydno, for example is sympatric with $H$. melpomene throughout much of its range and is today recognized as a separate species. In other cases, as in the forms of H. numata (Brown and Benson, 1974; Joron et al., this issue), separate species recognized by Eltringham have been united within a single polymorphic species. Various experiments with birds and lizards have confirmed the unpalatability of all Heliconius tested, as well as the related Laparus (Brower et al., 1963; Boyden, 1976; Chai, 1986, 1990; Pinheiro, 1996), and cyanogenic glycosides have been identified as key defensive compounds (Engler et al., 2000). Field studies have shown that natural selection towards the local mimicry ring is intense, as expected under Müllerian selection (Benson, 1972; Mallet and Barton, 1989; Mallet et al., 1990, 1998a; Kapan, 2001). Together, these data confirm the unpalatability and efficacy of mimicry to actual predators.

Yet in spite of the explosion of recent work, and his own diffidence about the value of his conclusions (see quotation at head of article), all of Eltringham's mimicry generalizations appear to hold. For example, more species in the melpomene group, particularly from the 'silvaniform' subgroup, go in for intergeneric mimicry than in the other two groups within Heliconius, and H. cydno is now known to mimic multiple species within the erato group (erato, sapho, eleuchia, and hewitsoni) as well as a member of another nymphalid subfamily (Elzunia humboldt in the Ithomiinae) (Linares, 1997a, b). Each of the erato-group models is itself mimicked mainly by only one or two other species in the melpomene group. Similarly, the multiple morphs of $H$. numata mimic multiple separate species of Melinaea and other ithomiines (Joron et al., this issue). The greater extra-heliconian mimicry of the melpomene group rather than the erato group, and the greater apparency of the erato group species also hold true. The existence of Eltringham's (1916) erato and melpomene groups as monophyletic entities has now been confirmed via mtDNA and nuclear DNA phylogenies, and the melpomene group appears more homogeneous in DNA sequence (Brower and Egan, 1997), implying a more recent origin than the erato group (9). 
Another argument that might be brought to bear is that the erato group are in general more gregarious than the melpomene group (6). Many of the species in the erato group have gregarious larvae, such as $H$. demeter, H. sapho, $H$. eleuchia, $H$. sara, while none of the $H$. melpomene group are gregarious (Brown, 1981). The gregarious species also pupate in clusters, and males visit these clusters and 'pupal-mate', that is mate with teneral females as they eclose (Deinert et al., 1994). The massed emergences together with pupal-mating males attracted from elsewhere provide a strong and highly visible display (5, 6). The melpomene group species neither pupate gregariously nor pupal-mate. In addition, the erato-group species roost gregariously in groups of up to about 20 at night. The melpomene group species also roost gregariously, but far more weakly, and often with the species they mimic rather than with members of their own species (Mallet, 1986a; Mallet and Gilbert, 1995).

(c) Polymorphism Perhaps surprisingly, polymorphism is fairly common in Heliconius and its allies. Since polymorphisms are expected of Batesian or quasi-Batesian diversifying frequency-dependent selection, and unexpected of simple Müllerian purifying selection, it is worth investigating cases of polymorphism in detail. There are many non-mimetic polymorphisms in limited zones of hybridization between races having distinct colour patterns (for example, between the pairs of races of erato and melpomene shown in Fig. 2; see Mallet et al., 1990). However, these narrow zones of polymorphism are clearly due to a balance between gene flow and disruptive mimetic selection on the pure races (Mallet et al., 1990, 1998a; Mallet, 1993). There are also a number of cases of more widespread polymorphism. The examples I treat here are H. cydno, H. numata, H. timareta, and Laparus doris. Three of these species are closely related members of the melpomene group, predominantly a group of mimics, as we have seen, even though this group contains among the most unpalatable species in the genus (Brower et al., 1963; Chai, 1986). In agreement with rule (10), these polymorphisms again make them more likely to be mimics than models.

Heliconius cydno copies multiple model species in the erato group and the unrelated Ithomiinae (see above), and this is as true within polymorphic populations as it is for different geographic races. In W. Ecuador, H. cydno alithea is polymorphic for yellow forms which mimic H. eleuchia primularis and white forms which mimic $H$. sapho candidus. The polymorphism is clinal, yellow forms being much commoner further south. This broad cline in $H$. cydno follows the distribution of its models, since $H$. sapho does not extend into Southern Ecuador, while $H$. eleuchia and $H$. sapho occur together further north. Recent field studies in W. Ecuador have shown that the direction of mimetic selection correlates with the relative abundances of the model species in the North and the South (Kapan, 2001). Dispersal and the relatively weak 
purifying selection expected when both types of mimics and both types of models are common (see Mallet and Joron, 1999) presumably causes the boundary between the two regimes to be broad, leading to widespread polymorphism. Similar processes explain the polymorphisms of $H$. cydno in Western Colombia, where different morphs mimic H. eleuchia eleusinus and $H$. sapho chocoensis to the West of the Westernmost Andes, H. eleuchia eleuchia, H. erato chestertonii and E. humboldt in the Cauca Valley (Linares, 1997a, b). In the central Cauca valley near Cali, forest clearance over the last two centuries has caused an expansion of $H$. erato chestertonii at the expense of $E$. humboldt. These vegetational and faunal changes have led to concomitant changes in the frequency of morphs within H. cydno. The frequency of form 'gustavi', the mimic of chestertonii, relative to the 'weymeri' mimic of Elzunia (Linares, 1997a) increased within H. cydno weymeri, giving excellent historical evidence of evolution brought about by the sort of spatially varying selection measured directly in Ecuador (Kapan, 2001). In the Dagua pass between the Cauca Valley and West coast of Colombia, three geographic races consisting of no less than five distinct mimetic forms meet: the yellow/white polymorphic $H$. cydno zelinde from the West, the 'gustavi'/"weymeri' forms of $H$. cydno weymeri from the Southern Cauca, and H. cydno cydnides from the Northern Cauca (Linares, 1997b).

The case of Heliconius numata, which may have up to ten morphs, each mimicking a different species of Melinaea (Ithomiinae), is similar. In a small area of N.E. Peru, there are strong differences locally in the abundance of Melinaea species, and morph frequencies of $H$. numata track this spatial variation of model abundance (Joron et al., this issue). Given its appropriate genetic architecture which allows for complex genetic switches to be achieved at a single locus, the polymorphism is readily explained by spatial (and possibly temporal) variation in relative model abundance (Brown and Benson, 1974; Joron et al., this issue), together with high rates of dispersal.

Polymorphism in $H$. timareta (Fig. 3) is harder to explain. This species, which may well be little more than a divergent subspecies of $H$. cydno (Brower, 1996; Brower and Egan, 1997) has up to four morphs on the Eastern slopes of the Andes in Ecuador. None of the morphs are mimetic, although all are brightly and apparently warningly coloured, and some forms resemble the rayed patterns of $H$. erato and co-mimics from the Amazonian areas nearby (however, rayed Heliconius co-mimics do not occur in the submontane sites from which $H$. timareta is best known). The species is a rare, narrow endemic, and little is known about its biology or palatability. In contrast, most of the other members of the $H$. cydno superspecies are strongly mimetic (see above). Possibly, H. timareta is a hybrid species whose polymorphism has been stabilized by chromosomal heterozygosity, as in paracentric inversions of Drosophila. The species remains enigmatic, but although it is polymorphic, it is also 

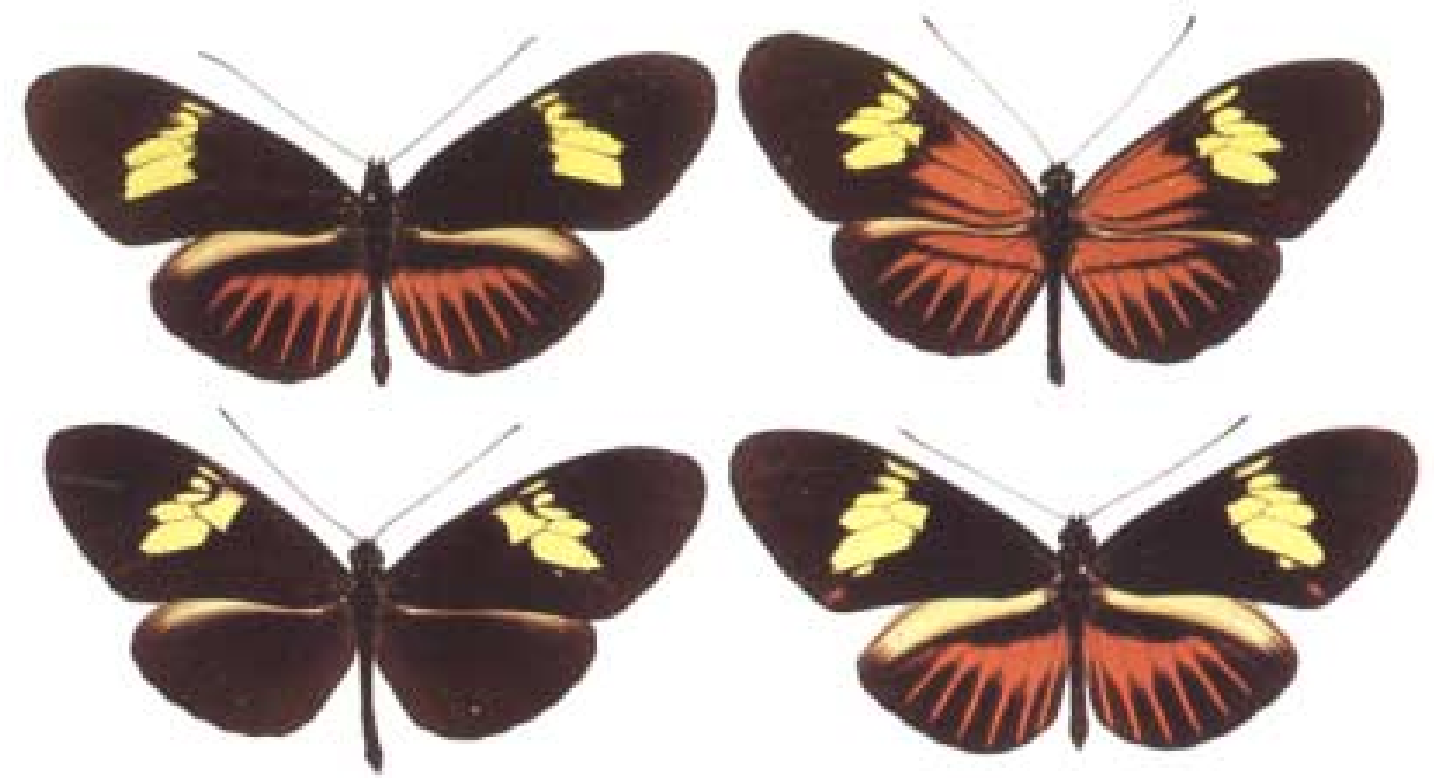

Figure 3. Polymorphism in Heliconius timareta. The figure shows four forms of $H$. timareta all from near Riobamba in Eastern Ecuador at approx. $1200 \mathrm{~m}$ alt. None of these forms appear to be mimetic, and they do not co-occur with forms that could be models; however, rayed Heliconius similar to the morph at top right (see Fig. 2) are common at lower elevations in the Amazon Basin of Ecuador and Peru. Photo (C) Bernard D'Abrera and the Natural History Museum. Reprinted from D'Abrera (1984), by permission.

completely non-mimetic and so cannot be a quasi-Batesian or Batesian mimic; instead, its conspicuous coloration suggests that timareta is as distasteful as its relatives in cydno and melpomene group.

The final polymorphisms treated here are in Laparus doris. This species has multiple mimetic morphs differing chiefly in the colour of the hindwing bar, which may be yellow, green, red-rayed, or blue (Fig. 4). Laparus is polymorphic virtually throughout tropical America. Brower et al. (1963) and Chai (1990) show this species to be unpalatable to birds, although somewhat less unpalatable than other Heliconius. This slightly greater palatability than its Heliconius models has led to the suggestion by Speed and Turner (1999) that L. doris is polymorphic as a result of quasi-Batesian mimicry. The mimicry of this species is relatively crude and impressionistic (8), which in addition to the polymorphism (10) suggests it is indeed the mimic. For example, the 'blue' morph is a tolerable mimic of $H$. sara and $H$. wallacei, but the dark blue iridescent sheen of these latter species are mimicked by a mixture of black and pale sky-blue scales in $L$. doris to make a dark bluish impression. The 'red' morph in the Amazon basin is a good mimic of the erato/melpomene rayed patterns in the area, but the effect is spoiled somewhat by the twinned yellow forewing bands $-H$. erato, $H$. melpomene and the majority of the rest of the rayed mimicry ring have only one (Figs. 2 and 4). The polymorphisms are highly clinal. A 'red' morph is common in Mexico, where it mimics species of 

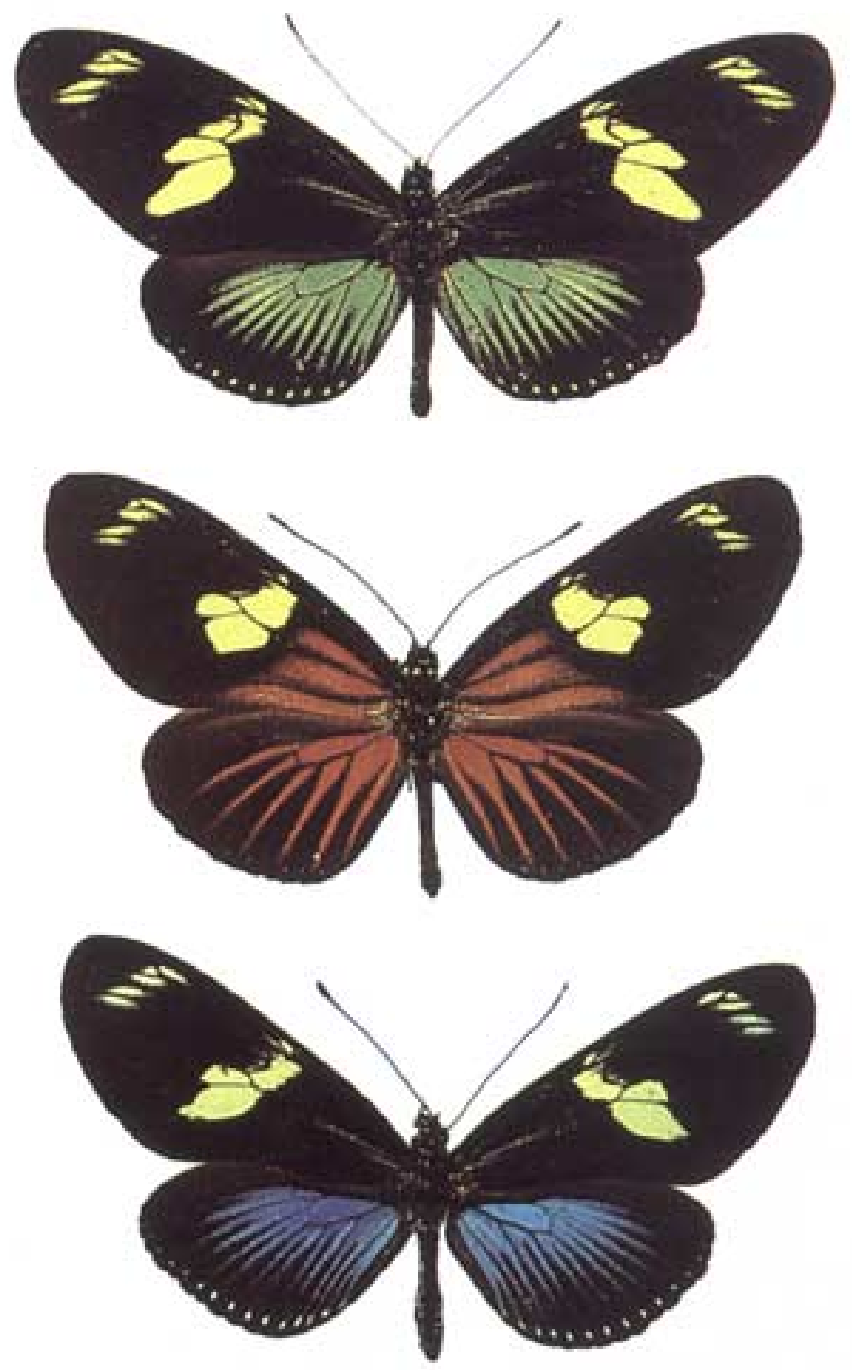

Figure 4. Polymorphic Müllerian mimicry in Laparus doris. The figure shows 'yellow' (from Southeastern Costa Rica), 'red' (from Eastern Ecuador; see also Fig. 2), and 'blue' forms of L. doris (from Western Ecuador). All forms are presumed Müllerian mimics, although darker versions of the 'yellow' form (known as the 'green' form) occur as rare non-mimetic variants throughout the range of the species. Photo $\odot$ Bernard D'Abrera and the Natural History Museum. Reprinted from D'Abrera (1984), by permission.

Parides, but rare further South in Central America, where the 'blue' saramimicking morphs predominate. In the Chiriquí area of Western Panama and adjacent Costa Rica, the 'yellow' morph becomes common, and is a clear mimic of the local H. hewitsoni, $H$. pachinus, and an unusual race of $H$. sara with a yellow-bordered hindwing. Elsewhere, the central part of the yellow bar 
of the 'yellow' morph appears at low frequency and with an increase in black scaling in the bar, making it look 'green', which is non-mimetic. This 'green' morph may be a recombinant between other morphs, but the genetics remain to be studied. In the savannahs of northeastern South America, the forewing bands are often white, like the local $H$. antiochus that acts as the model. Finally, the 'red' morph becomes commoner again in the Amazon basin, where it mimics the rayed mimicry ring (Figs. 2 and 4). This clinal variation suggests that spatial variation in mimetic selection, coupled with strong powers of dispersal, may maintain the polymorphisms, just as it maintains polymorphisms in H. cydno and H. numata.

In general, Laparus is rarer than its models (2), but this is not always true, because its populations fluctuate strongly. Caged bird experiments show it to be less unpalatable than most of the other species of Heliconius, but it is still strongly unpalatable and seems likely to be effectively unpalatable in the field: firstly, it has an unpleasant greasy appearance, a strong smell, and tends to feign death, as is typical of other highly unpalatable species; secondly, it has 'supergregarious' black-and-yellow-striped larvae which can be in many thousands on a single canopy vine of its Passiflora hostplant; L. doris may be the butterfly species with the most gregarious larvae. To ensure that so many larvae coexist, multiple females must lay eggs cooperatively on single shoots (Mallet and Jackson, 1980). When full-grown, these larvae descend to the base of the vine to pupate in tightly bunched groups of up to over a thousand. As the pupae hatch, males are attracted to the teneral females, and mate with them on the pupae or nearby. There may be many rotting pupae, as well as parasitoids, hyperparasitoids and sarcophagid flies present at the same time, making the immediate area stink of the bitter smell of these butterflies, their exuviae and meconium, as well as rotting insect flesh, while large numbers of a brightly coloured and normally rare butterfly species are flying round the pupae, perched in the vicinity, or mating over a period of a week or two. It is an impressive sight to anyone who has witnessed it. I imagine this conspicuous super-gregarious larval biology and resultant adult sexual aggregations would be impossible to maintain in the face of predator pressure unless the butterfly was as highly unpalatable as it appears. In conclusion, while it is possible that polymorphism in this species is due to quasi-Batesian mimicry (Speed, 1993; Speed and Turner, 1999), it seems more probable to me that $L$. doris is a highly unpalatable, classical Müllerian mimic under field conditions. As is true for the other species showing mimetic polymorphism, the polymorphism in L. doris clearly has a great deal to do with spatial variation in selection, overlapping ranges of model species, and a tendency to disperse long distances; it cannot necessarily be ascribed to quasi-Batesian mimicry and weak unpalatability.

In conclusion, while $H$. timareta remains anomalous, the mimetic polymorphisms in Heliconius are similar to those occurring in hybrid zones, in 
that all are clinal. The chief difference is that, in the polymorphic species treated here, bands of mimetic polymorphism can be much broader because of a suitable genetic architecture so that all morphs are mimetic, and because model species overlap in their distributions. In narrow hybrid zones between races of $H$. erato or H. melpomene, the polymorphisms are non-mimetic, and racial forms are strongly selected against on the wrong sides of the hybrid zones (Mallet and Barton, 1989). There is no convincing evidence for quasiBatesian mimicry in any of these species.

(d) Heliconius erato and melpomene Perhaps the most difficult mimics to evaluate, and also the most likely to coevolve, are H. erato and H. melpomene themselves. These two species are among the best-studied neotropical organisms, and their colour patterns have diversified geographically to an extraordinary extent (Brown et al., 1974; Brown, 1979; Sheppard et al., 1985; Mallet, 1993; Turner and Mallet, 1996). It is well known that each race of $H$. erato has an equivalent co-mimic within $H$. melpomene, except in a very few cases. The mimicry is so exact and the biology of the two species so similar, that opinions differ on which is the mimic and which the model. Eltringham (1916) cites the greater abundance of erato in most areas as an indication of that species being the model; however, he points out that $H$. melpomene xenoclea is more common than its co-mimic $H$. erato microclea in collections from Peru. Keith Brown (pers. comm.) also argues that in some sites on the Eastern slopes of the Andes H. melpomene outnumbers H. erato and may sometimes act as the model. However, based my own experience in Mexico, Costa Rica (see also Gilbert, 1984), Panama (Mallet, 1986b), Colombia (Mallet and Jackson, 1980), Ecuador (Jiggins et al., 1996), Peru (Mallet et al., 1990), Venezuela and Guyana, this is almost never the case whatever the habitat, except in a very few localities where melpomene larval hostplants are unusually common: H. erato is almost always over twice as abundant as $H$. melpomene. Furthermore, H. erato penetrates into drier, more disturbed and more marginal habitats than $H$. melpomene, such as in isolated cloud forest at the northern end of the desert in the Guajira peninsula of Colombia (Mallet, 1993). Heliconius erato is also more widely distributed geographically: it occurs throughout Mexico, while H. melpomene enters only the southern part of Central America. Heliconius erato also occurs throughout much of Argentina and Paraguay, again a long way further south than H. melpomene (Brown, 1979).

Brown et al. (1974), Sheppard et al. (1985) and especially Turner (1984, see also Turner and Mallet, 1996), have frequently argued that the divergence of $H$. erato and H. melpomene into diverse geographic races took place as a result of mimetic adaptation to mimicry rings including many other species in Pleistocene refugia, which were thought to coincide with temperate-zone ice ages. One problem with this hypothesis is that the putative models, including 
pierids and other heliconiines which these authors suggest caused the divergence, are always rarer than their supposed mimics. In many extra-Amazonian locations, particularly in the Andes and further West, there are simply no good candidates for models of the paired races of Heliconius erato and melpomene (for example, their patterns at the top of Fig. 2); indeed there are no other obvious mimics at all. In conclusion, it is most likely that one of these two species is the major model for rarer mimics, rather than the other way round (Mallet, 1993). Because H. erato is almost always the most apparent, gregarious, and widespread of the two, and because, where patterns differ (e.g. nailhead rays) other mimics clearly copy erato rather than melpomene. It is almost certainly erato that acts as the model.

While mimicry has probably been mostly via advergence of melpomene to erato, it is still possible that local conditions occasionally allow the reverse evolutionary route for some geographic races as a result of 'alternating' coevolution (Marshall, 1908). Gilbert (1983) agreed that melpomene normally mimics erato throughout its range, but he felt there was some evidence for advergence by erato to melpomene in Costa Rica. In the absence of melpomene, the Mexican form of $\mathrm{H}$. erato has a very narrow yellow hindwing bar. When this species comes into sympatry with melpomene further south, the erato bar becomes broader, in line with the typical breadth of the hindwing bar of the local melpomene. However, this example is not, to my mind, completely clear; the H. melpomene pattern could simply be copying the local version of the erato pattern which happens to have diverged independently of H. melpomene. Given the massive geographic diversification of colour patterns in both of these species, variation in the breadth of yellow bar seems trivial by comparison.

Two other cases seem to some to indicate some evolutionary adaptation of erato to other species. Sheppard et al. (1985) suggested that $H$. erato and $H$. melpomene in West Ecuador uniquely developed white hindwing fringes in partial mimicry of the local geographic races of $H$. sara, $H$. cydno and $H$. sapho (even though the rest of the pattern remained distinct). However, these species also have white hindwing fringes in other areas where erato does not copy this feature, and the fringes are far more similar between $\mathrm{H}$. erato and $\mathrm{H}$. melpomene than between these and any of the other species. While potentially true, the argument for erato being a mimic again lacks force. A final example is in the Cauca valley, where $H$. melpomene is absent, and $H$. erato chestertonii is said to converge towards the pattern of $H$. cydno form 'gustavi' (Turner, 1976). The pattern, which lacks the forewing band found in all other races, is certainly unusual by erato standards. Some facts again point to the notion that cydno is here mimicking erato rather than vice-versa. Firstly, chestertonii occurs throughout the Cauca Valley, whereas 'gustavi', usually classified as part of the polymorphic subspecies $H$. cydno weymeri (Brown, 1979), occurs commonly only in the southern portion of the valley. Secondly, an analysis of historical 
collections shows that habitat disturbance led to $H$. erato chestertonii becoming more abundant, and has been accompanied by an increase in the frequency of the 'gustavi' morph of cydno (see above, and Linares, 1997a). Thirdly, chestertonii appears to be more ancient than most other races of erato, and especially than its co-mimic 'gustavi', which exists as a polymorphic intraracial form. The mtDNA divergence of the latter is weak, whereas the former is strongly divergent from the rest of erato, comparable with another major erato clade now normally considered a separate species, H. himera (Brower, 1996). Indeed, the scarcity of hybrids between $H$. erato chestertonii and $H$. erato venus in the Dagua Pass of W. Colombia where they abut suggests that chestertonii acts as an occasionally hybridizing separate species rather than as a freely compatible geographic race of erato (M. Linares and C.D. Jiggins, pers. comm.). Heliconius himera and $H$. erato interact similarly in Ecuador and Northern Peru (Jiggins et al., 1996; Mallet et al., 1998b). The divergence in cydno is at a more trivial taxonomic and DNA level than in erato, suggesting a later, mimetic origin of the 'gustavi' pattern (see above, and Brower, 1996). The idea that erato chestertonii evolved to mimic cydno therefore seems untenable.

While the literature suggests that erato might sometimes mimic melpomene or other species in some areas, rather than the always other way round, there is no very convincing evidence proving that this is the case. Most of the examples also agree as well or better with a null hypothesis of no coevolution at all, even of a geographically alternating kind, such that erato is the model throughout its range.

\section{Conclusions}

Müllerian mimicry should often be a mutualism, so we might naively expect to see evidence for mutual evolutionary convergence. However, empirical and comparative evidence for mutual convergence is weak or non-existent. A possible route to coevolution could occur if a species is an abundant model in one area and a rare mimic in another. This would be an example of species level coevolution caused by a geographic mosaic of unilateral mimicry in opposite directions between local populations (see also Marshall, 1908; Thompson, 1994). But there is no good evidence even for this limited type of coevolution. Nevertheless, the abundant evidence for unidirectional mimicry evolution should not be taken as strong evidence that the mimicry is parasitic or quasiBatesian, because one-sided approach and mimetic polymorphisms can also be a normal outcome of mutualistic Müllerian mimicry. Theory shows that inequalities in protection between two unpalatable species are evolutionarily amplified to produce Batesian-like evolution of a less protected mimic towards a more strongly protected model, even though mutualism is achieved when 
mimicry is complete. Polymorphisms can arise easily due to spatially varying selection on true Müllerian mimics, and this has now been demonstrated empirically for two major polymorphic species of Heliconius (Linares, 1997a; Kapan, 2001; Joron et al., this issue). Further work is needed to investigate whether the theoretically possible quasi-Batesian or mutually convergent, coevolutionary Müllerian mimicry can ever occur in nature. I challenge future workers to find better evidence for either!

\section{Acknowledgments}

I am grateful to Keith Brown, Mathieu Joron, Gerardo Lamas, Mike Speed, John Turner, and an anonymous reviewer for ongoing discussions and comments on the manuscript. I thank Paul Williams and Ian Wynne for comments on the bumble bee evidence. I am particularly grateful to Mike Speed, as I realized that the extension of Müller's theory for variable unpalatability (see Appendix) would be a useful contribution while reviewing his alternative ideas for this issue; the analysis benefited from discussions with Russ Naisbit, Andrew Pomiankowski and Ziheng Yang. This work was supported by grants from NERC, BBSRC, and the British Council.

\section{Appendix: Extending Müller's result for unequal unpalatabilities}

Müller's (1879) argument for the evolutionary advantages of mimicry between unpalatable species contains perhaps the first quantitative model of Darwinian relative fitness. For comparative purposes, the argument used here is as similar as possible to Müller's. Again, there are two species; Müller's assumption that a number $n$ must be 'destroyed' in a 'summer' to effect learning by local predators is modified for unequal palatabilities so that species 1 , if alone, loses $n_{1}$ individuals, while species 2 , if alone, loses $n_{2}$ individuals.

These assumptions are justifiable if we imagine that there is a threshold dose $D$ of unpalatable compounds per unit time that results in avoidance, and that the species differ in the per capita dosages $d_{1}$ and $d_{2}$ of some compound. The actual relationship between dose received by predators and the probability of attack will be some sort of sigmoid curve rather than the step function assumed in this simple Müllerian formulation (Fig. 5), where $D$ represents the inflection point, or $A D_{50}$ (the dose received at which the attack probability is about $50 \%$ of maximal). If the dose received is $<<D$, the probability of attack is approximately maximal; if the dose received $>>$, the attack probability is approximately zero.

These assumptions imply that, when each species is distinct:

$$
\begin{aligned}
& D=n_{1} d_{1} \\
& D=n_{2} d_{2}
\end{aligned}
$$

As an aside, it is worth noting that the degrees of unpalatability or dosage $d_{1}$ and $d_{2}$ are inversely proportional to the Müllerian parameters $n_{1}$ and $n_{2}$, because $d_{i}=D / n_{i}$. Thus the Müllerian parameters $n_{i}$ effectively measure 'palatability'; in the limit, when the dosage $d_{i}$ is zero, an infinite number of prey must be eaten for avoidance to be learnt. 


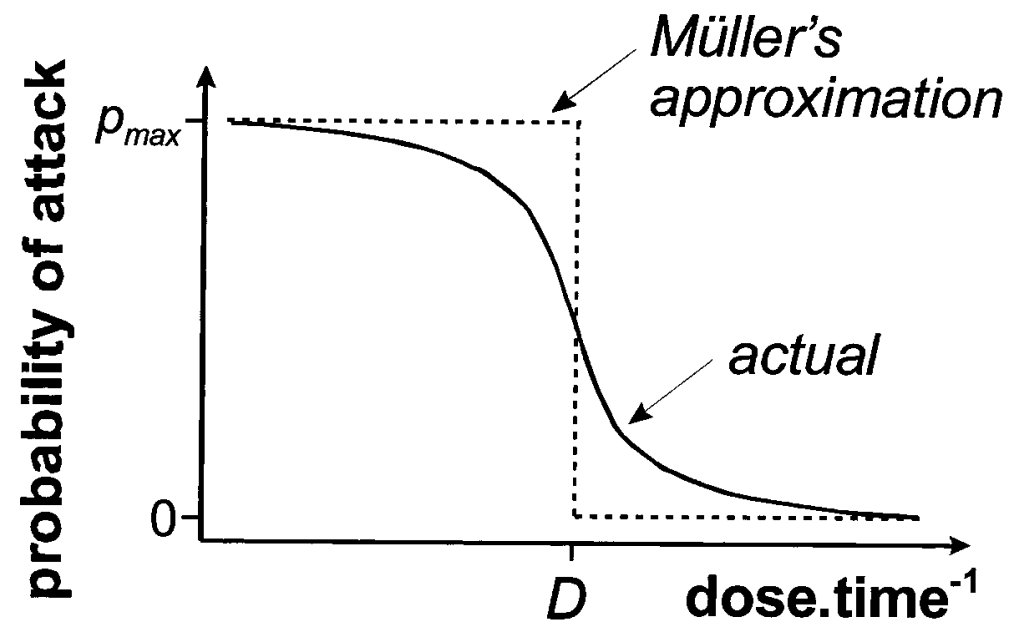

Figure 5. Dose-response curve for probability of attack, and Müller's approximation.

Combining (1) and (2):

$$
\frac{n_{1}}{n_{2}}=\frac{d_{2}}{d_{1}}
$$

When the two species are morphologically indistinguishable mimics, both contribute to the total dose:

$$
D=l_{1} d_{1}+l_{2} d_{2}
$$

where $l_{1}$ and $l_{2}$ represent the numbers of individuals lost per unit time (in Müller's terms, 'during one summer') by each prey species during predator learning of the mimetic pair.

A second assumption we will make (again following Müller) is that, if the two species are indistinguishable to the predators before tasting, the numbers lost during predator learning will be directly proportional to their overall abundances $a_{1}$ and $a_{2}$. Thus:

$$
\frac{l_{1}}{l_{2}}=\frac{a_{1}}{a_{2}}
$$

We are interested in obtaining the values of $l_{1}$ and $l_{2}$ in terms of the abundances of each species $a_{1}$ and $a_{2}$ and the Müllerian palatability parameters $n_{1}$ and $n_{2}$. From (1) and (4) we obtain:

$$
l_{1} d_{1}+l_{2} d_{2}=n_{1} d_{1}
$$

Rearranging and substituting from (3) and (5):

$$
l_{1}=\frac{a_{1} n_{1} n_{2}}{a_{1} n_{2}+a_{2} n_{1}}
$$

Similarly:

$$
l_{2}=\frac{a_{2} n_{1} n_{2}}{a_{1} n_{2}+a_{2} n_{1}}
$$


Now, following Müller's arguments, we find the total benefit, in terms of extra individuals surviving, for species 1 when it mimics species 2 :

$$
n_{1}-l_{1}=n_{1}-\frac{a_{1} n_{1} n_{2}}{a_{1} n_{2}+a_{2} n_{1}}=\frac{a_{2} n_{1}^{2}}{a_{1} n_{2}+a_{2} n_{1}}
$$

Similarly, the total benefit for species 2 is:

$$
n_{2}-l_{2}=\frac{a_{1} n_{2}^{2}}{a_{1} n_{2}+a_{2} n_{1}}
$$

The per capita fitness gains, $g_{1}$ and $g_{2}$ for each species are:

$$
g_{1}=\frac{a_{2} n_{1}^{2}}{a_{1}\left(a_{1} n_{2}+a_{2} n_{1}\right)} \quad \text { and } \quad g_{2}=\frac{a_{1} n_{2}^{2}}{a_{2}\left(a_{1} n_{2}+a_{2} n_{1}\right)} .
$$

In this formulation, therefore, $g_{1}$ and $g_{2}$ are always positive: provided the two species are unpalatable as assumed here, it is always somewhat advantageous for both species to be part of a mimicry ring, even if one species differs greatly in unpalatability from another.

The relative fitness gains are thus:

$$
\frac{g_{1}}{g_{2}}=\frac{a_{2}^{2}}{a_{1}^{2}} \frac{n_{1}^{2}}{n_{2}^{2}}
$$

This is similar to Müller's (1879) result. The relative gains are in proportion to the square of the relative palatabilities, as well as in proportion to the square of the relative population densities of each species. It makes sense, of course, that the result applies to unpalatability as well as relative population density, because the total dosage the predator receives was assumed linear with the numbers of individuals tasted; dosage per prey and prey numbers are therefore expected to act in the same way on the relative benefits of mimicry.

Müller's result (and also this extended version) is particularly interesting in the context of this paper in that differences in abundance and unpalatability cause the benefits of mimicry for rarer or less unpalatable mimics to be amplified - squared - compared to what might be expected from density and unpalatability differences alone.

\section{References}

Ackery, P.R. and Vane-Wright, R.I. (1984) Milkweed Butterflies: Their Cladistics and Biology. British Museum, Natural History, London.

Bates, H.W. (1862) Contributions to an insect fauna of the Amazon valley (Lepidoptera: Heliconidae). Trans. Linn. Soc. Lond. 23, 495-566.

Bates, H.W. (1879) [Commentary on Müller's paper]. Trans. Entomol. Soc. Lond. 1879, xxviii-xxix. Benson, W.W. (1972) Natural selection for Müllerian mimicry in Heliconius erato in Costa Rica. Science 176, 936-939.

Boyden, T.C. (1976) Butterfly palatability and mimicry: experiments with Ameiva lizards. Evolution 30, 73-81.

Brower, A.V.Z. (1996) Parallel race formation and the evolution of mimicry in Heliconius butterflies: a phylogenetic hypothesis from mitochondrial DNA sequences. Evolution 50, 195-221.

Brower, A.V.Z. and Egan, M.G. (1997) Cladistic analysis of Heliconius butterflies and relatives (Nymphalidae: Heliconiiti): a revised phylogenetic position for Eueides based on sequences from mtDNA and a nuclear gene. Proc. Roy. Soc. Lond. B 264, 969-977.

Brower, J.V.Z. (1958) Experimental studies of mimicry in some North American butterflies. Part I. The monarch, Danaus plexippus, and viceroy, Limenitis archippus archippus. Evolution 12, 32-47. 
Brower, L.P. (1984) Chemical defence in butterflies. In R.I. Vane-Wright and P.R. Ackery (eds) The Biology of Butterflies. Academic Press, London (Symposia of the Royal Entomological Society of London; 11), pp. 109-134.

Brower, L.P., Brower, J.V.Z. and Collins, C.T. (1963) Experimental studies of mimicry. 7. Relative palatability and Müllerian mimicry among Neotropical butterflies of the subfamily Heliconiinae. Zoologica, N.Y. 48, 65-84.

Brown, K.S. (1979) Ecologia Geográfica e Evolução nas Florestas Neotropicais. Universidade Estadual de Campinas, Campinas, Brazil, Livre de Docencia.

Brown, K.S. (1981) The biology of Heliconius and related genera. Ann. Rev. Entomol. 26, 427-456.

Brown, K.S. and Benson, W.W. (1974) Adaptive polymorphism associated with multiple Müllerian mimicry in Heliconius numata (Lepid.: Nymph.). Biotropica 69, 205-228.

Brown, K.S., Sheppard, P.M. and Turner, J.R.G. (1974) Quaternary refugia in tropical America: evidence from race formation in Heliconius butterflies. Proc. Roy. Soc. Lond. B 187, 369-378.

Chai, P. (1986) Field observations and feeding experiments on the responses of rufous-tailed jacamars (Galbula ruficauda) to free-flying butterflies in a tropical rainforest. Biol. J. Linn. Soc. 29, 166-189.

Chai, P. (1990) Relationships between visual characteristics of rainforest butterflies and responses of a specialized insectivorous bird. In M. Wicksten (ed.) Adaptive Coloration in Invertebrates. Texas A\&M University Sea Grant College Program, College Station, TX, USA (Proceedings of a Symposium Sponsored by the American Society of Zoologists), pp. 31-60.

D'Abrera, B. (1984) Butterflies of the Neotropical Region, Vol., Part II. Danaidae, Ithomiidae, Heliconidae \& Morphidae. Hill House, Ferny Creek, Victoria, Australia.

Deinert, E.I., Longino, J.T. and Gilbert, L.E. (1994) Mate competition in butterflies. Nature 370, 23-24.

Dixey, F.A. (1907) Recent developments in the theory of mimicry. Proc. Brit. Assoc. 1907, 1-2.

Dixey, F.A. (1909) On Müllerian mimicry and diaposematism. Trans. Entomol. Soc. Lond. 1908, 559-583.

Edmunds, M. (1974) Defence in Animals. Longmans, Harlow, Essex.

Eltringham, H. (1916) On specific and mimetic relationships in the genus Heliconius. Trans. Entomol. Soc. Lond. 1916, 101-148.

Emsley, M.G. (1964) The geographical distribution of the color-pattern components of Heliconius erato and Heliconius melpomene with genetical evidence for the systematic relationship between the two species. Zoologica, N.Y. 49, 245-286.

Emsley, M.G. (1965) Speciation in Heliconius (Lep., Nymphalidae): morphology and geographic distribution. Zoologica, N.Y. 50, 191-254.

Engler, H., Spencer, K.C. and Gilbert, L.E. (2000) Preventing cyanide release from leaves. Nature 406, 144-145.

Futuyma, D.J. (1998) Evolutionary Biology. Sinauer, Sunderland, Mass.

Gilbert, L.E. (1983) Coevolution and Mimicry. In D.J. Futuyma and M. Slatkin (eds) Coevolution. Sinauer Associates, Sunderland, Mass, pp. 263-281.

Gilbert, L.E. (1984) The biology of butterfly communities. In R.I. Vane-Wright and P.R. Ackery (eds) The Biology of Butterflies. Academic Press, London (Symposia of the Royal Entomological Society of London; 11), pp. 41-54.

Huheey, J.E. (1988) Mathematical models of mimicry. Am. Nat. 131, S22-S41.

Jiggins, C.D., McMillan, W.O., Neukirchen, W. and Mallet, J. (1996) What can hybrid zones tell us about speciation? The case of Heliconius erato and H. himera (Lepidoptera: Nymphalidae). Biol. J. Linn. Soc. 59, 221-242.

Joron, M. and Mallet, J. (1998) Diversity in mimicry: paradox or paradigm? Trends Ecol. Evol. 13, 461-466.

Kapan, D. (2000) Three-butterfly system provides a field test of Müllerian mimicry. Nature 409, $338-340$.

Linares, M. (1997a) The ghost of mimicry past: laboratory reconstitution of an extinct butterfly 'race'. Heredity 78, 628-635. 
Linares, M. (1997b) Origin of neotropical mimetic diversity from a three-way hybrid zone of Heliconius cydno butterflies. In H. Ulrich (ed.) Tropical Diversity and Systematics. Zoologisches Forschunginstitut und Museum Alexander Koenig, Bonn. (Proceedings of the International Symposium on Biodiversity and Systematics in Tropical Ecosystems, Bonn, 1994), pp. 93-108.

Mallet, J. (1986a) Gregarious roosting and home range in Heliconius butterflies. Natl. Geogr. Res. 2, 198-215.

Mallet, J. (1986b) Hybrid zones in Heliconius butterflies in Panama, and the stability and movement of warning colour clines. Heredity 56, 191-202.

Mallet, J. (1993) Speciation, raciation, and color pattern evolution in Heliconius butterflies: evidence from hybrid zones. In R.G. Harrison (ed.) Hybrid Zones and the Evolutionary Process. Oxford University Press, New York, pp. 226-260.

Mallet, J. and Barton, N.H. (1989) Strong natural selection in a warning color hybrid zone. Evolution 43, 421-431.

Mallet, J. and Gilbert, L.E. (1995) Why are there so many mimicry rings? Correlations between habitat, behaviour and mimicry in Heliconius butterflies. Biol. J. Linn. Soc. 55, 159-180.

Mallet, J. and Jackson, D.A. (1980) The ecology and social behaviour of the Neotropical butterfly Heliconius xanthocles Bates in Colombia. Zool. J. Linn. Soc. 70, 1-13.

Mallet, J. and Joron, M. (1999) The evolution of diversity in warning colour and mimicry: polymorphisms, shifting balance, and speciation. Ann. Rev. Ecol. Syst. 30, 201-233.

Mallet, J., Barton, N., Lamas, G., Santisteban, J., Muedas, M. and Eeley, H. (1990) Estimates of selection and gene flow from measures of cline width and linkage disequilibrium in Heliconius hybrid zones. Genetics 124, 921-936.

Mallet, J., Jiggins, C.D. and McMillan, W.O. (1996) Mimicry meets the mitochondrion. Current Biol. 6, 937-940.

Mallet, J., McMillan, W.O. and Jiggins, C.D. (1998a) Mimicry and warning color at the boundary between races and species. In D.J. Howard and S.H. Berlocher (eds) Endless Forms: Species and Speciation. Oxford University Press, New York, pp. 390-403.

Mallet, J., McMillan, W.O. and Jiggins, C.D. (1998b) Estimating the mating behavior of a pair of hybridizing Heliconius species in the wild. Evolution 52, 503-510.

Marshall, G.A.K. (1908) On diaposematism, with reference to some of the limitations of the Müllerian hypothesis of mimicry. Trans. Entomol. Soc. Lond. 1908, 93-142.

Müller, F. (1879) Ituna and Thyridia; a remarkable case of mimicry in butterflies. Trans. Entomol. Soc. Lond. 1879, xx-xxix.

Nicholson, A.J. (1927) A new theory of mimicry in insects. Austral. Zool. 5, 10-104.

Owen, R.E. and Owen, A.R.G. (1984) Mathematical paradigms for mimicry: recurrent sampling. J. Theoret. Biol. 109, 217-247.

Pinheiro, C.E.G. (1996) Palatability and escaping ability in neotropical butterflies: tests with wild kingbirds (Tyrannus melancholicus). Biol. J. Linn. Soc. 59, 351-365.

Platt, A.P. (1983) Evolution of North American admiral butterflies (Limenitis: Nymphalidae). Bull. Entomol. Soc. Amer. 29, 10-22.

Plowright, R.C. and Owen, R.E. (1980) The evolutionary significance of bumble bee color patterns: a mimetic interpretation. Evolution 34, 622-637.

Ricklefs, R.E. and Miller, G.L. (2000) Ecology. Freeman, New York.

Ritland, D.B. (1990) Localized interspecific hybridization between mimetic Limenitis butterflies (Nymphalidae) in Florida. J. Lepid. Soc. 44, 163-173.

Ritland, D.B. (1991) Revising a classic butterfly mimicry scenario: demonstration of Müllerian mimicry between Florida viceroys (Limenitis archippus floridensis) and queens (Danaus gilippus berenice). Evolution 45, 918-934.

Ritland, D.B. and Brower, L.P. (1991) The viceroy butterfly is not a Batesian mimic. Nature 350, 497-498.

Sheppard, P.M., Turner, J.R.G., Brown, K.S., Benson, W.W. and Singer, M.C. (1985) Genetics and the evolution of muellerian mimicry in Heliconius butterflies. Philos. Trans. Royal Soc. Lond. B 308, 433-613. 
Speed, M.P. (1993) Muellerian mimicry and the psychology of predation. Anim. Behav. 45, 571580.

Speed, M.P. (1999) Robot predators in virtual ecologies: the importance of memory in mimicry studies. Anim. Behav. 57, 203-213.

Speed, M.P. and Turner, J.R.G. (1999) Learning and memory in mimicry: II. Do we understand the mimicry spectrum? Biol. J. Linn. Soc. 67, 281-312.

Speed, M.P., Alderson, N.J., Hardman, C. and Ruxton, G.D. (2000) Testing Mullerian mimicry: an experiment with wild birds. Proc. Roy. Soc. Lond. B 267, 725-731.

Thompson, J.N. (1994) The Coevolutionary Process. Chicago University Press, Chicago.

Turner, J.R.G. (1976) Muellerian mimicry: classical "beanbag" evolution and the role of ecological islands in adaptive race formation. In S. Karlin and E. Nevo (eds) Population Genetics and Ecology. Academic Press, New York, pp. 185-218.

Turner, J.R.G. (1977) Butterfly mimicry - the genetical evolution of an adaptation. Evol. Biol. 10, $163-206$.

Turner, J.R.G. (1978) Why male butterflies are non-mimetic: natural selection, sexual selection, group selection, modification and sieving. Biol. J. Linn. Soc. 10, 385-432.

Turner, J.R.G. (1984) Mimicry: the palatability spectrum and its consequences. In R.I. VaneWright and P.R. Ackery (eds) The Biology of Butterflies. Academic Press, London (Symposia of the Royal Entomological Society of London; 11), pp. 141-161.

Turner, J.R.G. (1995) Mimicry as a model for coevolution. In R. Arai, M. Kato and Y. Doi (eds) Biodiversity and Evolution. National Science Museum Foundation, Tokyo, pp. 131-150.

Turner, J.R.G. and Mallet, J.L.B. (1996) Did forest islands drive the diversity of warningly coloured butterflies? Biotic drift and the shifting balance. Philos. Trans. Royal Soc. Lond. B 351, $835-845$.

Waldbauer, G.P. (1988) Asynchrony between Batesian mimics and their models. Am. Nat. 131, s103-s121.

Wickler, W. (1968) Mimicry in Plants and Animals. McGraw-Hill, New York.

Williams, P.H. (1985) A preliminary cladistic investigation of relationships among the bumble bees (Hymenoptera: Apidae). Syst. Entomol. 10, 239-255.

Williams, P.H. (1991) The bumble bees of the Kashmir Himalaya (Hymenoptera: Apidae, Bombini). Bull. Brit. Mus. (Nat. Hist.), Entomol. 60, 1-204.

Williams, P.H. (1994) Phylogenetic relationships among bumble bees (Bombus Latr.): a reappraisal of morphological evidence. Syst. Entomol. 19, 327-344. 